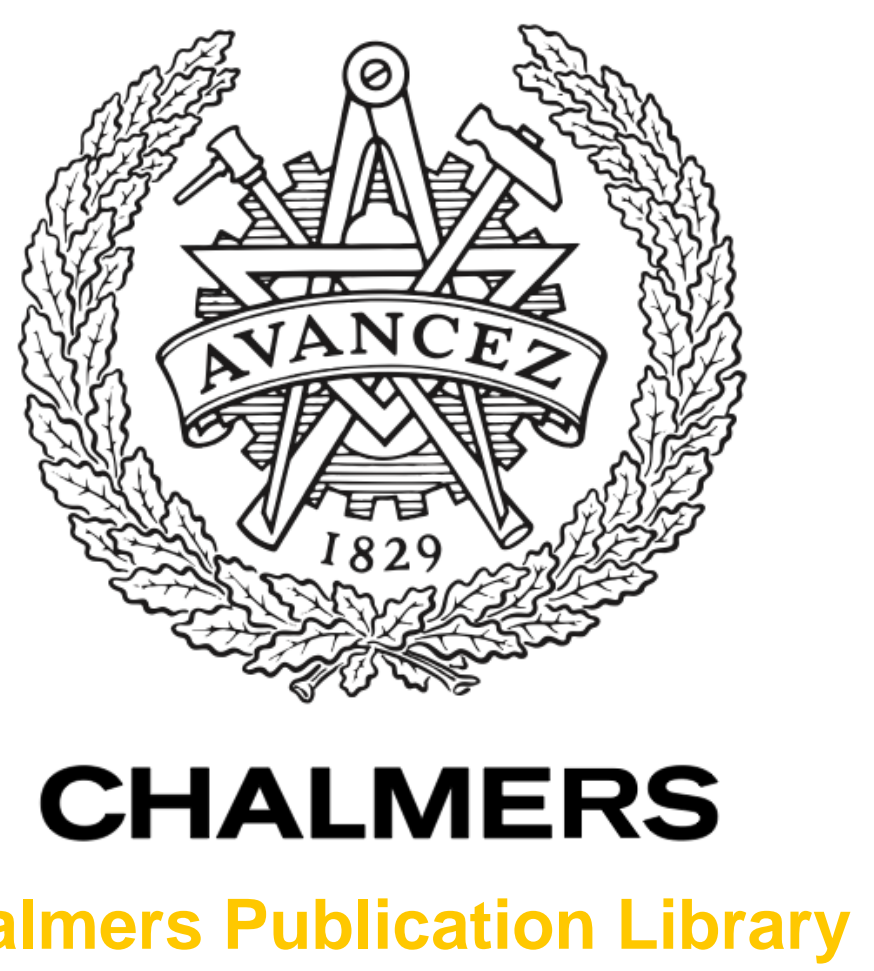

Supercontinuum generation in silicon waveguides based on optical wave-breaking

This document has been downloaded from Chalmers Publication Library (CPL). It is the author's version of a work that was accepted for publication in:

\title{
Nonlinear Photonics
}

Citation for the published paper:

Castello-Lurbe, D. ; Torres Company, V. ; Silvestre, E. (2014) "Supercontinuum generation in silicon waveguides based on optical wave-breaking". Nonlinear Photonics

Downloaded from: http://publications.lib.chalmers.se/publication/201121

Notice: Changes introduced as a result of publishing processes such as copy-editing and formatting may not be reflected in this document. For a definitive version of this work, please refer to the published source. Please note that access to the published version might require a subscription. 


\title{
Supercontinuum generation in silicon waveguides based on optical wave-breaking
}

\author{
David Castelló-Lurbe ${ }^{1, *}$, Victor Torres-Company ${ }^{2}$, and Enrique Silvestre ${ }^{1}$ \\ ${ }^{1}$ Departament d'Òptica, Universitat de València, 46100 Burjassot, Spain \\ ${ }^{2}$ Microtechnology and Nanoscience Department (MC2), Chalmers University of Technology, 41296 Göteborg, \\ Sweden \\ *david.castello-lurbe@uv.es
}

\begin{abstract}
We theoretically find the third order dispersion that optimizes the spectral broadening induced by optical wave-breaking. It produces supercontinuum spectra spanning beyond $2 / 3$ of an octave in a silicon waveguide pumping at $1550 \mathrm{~nm}$.
\end{abstract}

OCIS codes: $130.4310,190.5530$.

\section{Introduction}

The silicon-on-insulator (SOI) platform offers great potential for on-chip light manipulation due to its strong thirdorder nonlinearities together with the tight optical confinement enabled by its high-index contrast [1]. Nevertheless, two-photon absorption (TPA) significantly limits the spectral broadening possibilities at telecom wavelengths [2].

Recently, dispersive waves emission in the normal dispersion regime has been demonstrated [3] and related to the so-called optical wave-breaking (OWB) [5]. Here we follow [4] to calculate the third-order dispersion (TOD) required to induce dispersive wave emission through OWB, whose theoretical location differs from the analysis carried out in [3]. The validity of our analysis is firstly confirmed in lossless media. In a second step, we succesfully apply this process on silicon waveguides to produce supercontinuum spectra even pumping at telecom wavelengths.

\section{Optical wave-breaking induced spectral broadening}

Following [4], where we introduced a generalized version of the length scales where self-phase modulation (SPM) and the group-velocity dispersion (GVD), respectively, act at each propagation distance, we derive the TOD required to achieve phase-matching between the waves located in the leading pulse tail and the corresponding resonant frequency induced by OWB

$$
\beta_{3}^{2}=\left(\sigma_{2}-\frac{\sigma_{3}}{3 \varepsilon}\right) \frac{\sqrt{2} \beta_{2}^{3}}{\varepsilon \gamma_{0} P_{0}}, \quad \text { and } \quad \omega_{\mathrm{res}}-\omega_{0}=\frac{-2 \beta_{2}}{\beta_{3}},
$$

where $\sigma_{k}=2 \int_{-\infty}^{0}\left(\partial_{x} U\right)^{k} U \mathrm{~d} x / \int_{-\infty}^{+\infty} U^{2} \mathrm{~d} x$, being $U$ the normalized input pulse power, $x=t / T_{0}$, where $T_{0}$ indicates the pulse temporal width, $\varepsilon$ represents the maximum of $\partial_{x} U, P_{0}$ is the input pulse peak power, $\gamma_{0}$ is the nonlinear coefficient, and $\beta_{i}$ corresponds to the dispersive coefficients evaluated at the carrier frequency, $\omega_{0}$. We note that the resonant condition is slightly different to the one derived in [3] due to the nonlinear mixing considered here.

In order to verify our results we study both the long and short pulse regime, always pumping at $1550 \mathrm{~nm}$. On the one hand, we consider $\beta_{2}=7.5 \mathrm{ps}^{2} \mathrm{~km}^{-1}, \gamma_{0}=2.5 \mathrm{~W}^{-1} \mathrm{~km}^{-1}$, a sech input pulse with $P_{0}=0.6 \mathrm{~kW}$ and $T_{0}=1 \mathrm{ps}$ (as in [3]),
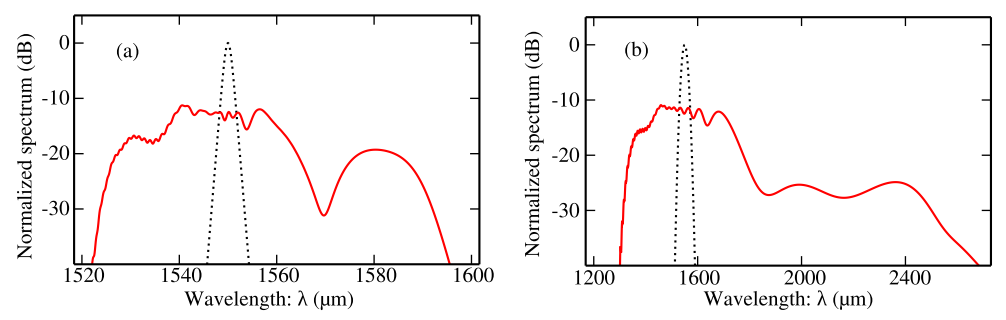

Fig. 1. Resonant wave emission via OWB in (a) long pulse (1 ps) and (b) short pulse (0.1 ps) regime. 

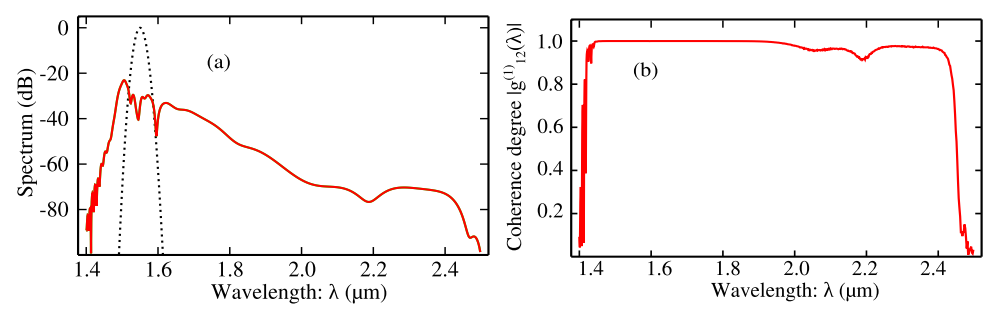

Fig. 2. (a) Input (dashed curve) and output (solid curve) pulse spectra. (b) Degree of coherence of the pulse ensemble.

from which we computed $\beta_{3}=0.5 \mathrm{ps}^{2} \mathrm{~km}^{-1}$ attending to Eq. (1). The output spectrum after $35 \mathrm{~m}$ is plotted in Fig. 1(a), where we observe a broadband resonance centered at $\lambda=1580 \mathrm{~nm}$ near $\lambda_{\text {res }}=1589 \mathrm{~nm}$, which was calculated through Eq. (1). On the other hand, in Fig. 1(b) we consider $\beta_{2}=0.2 \mathrm{ps}^{2} \mathrm{~m}^{-1}, \gamma_{0}=4.0 \mathrm{~W}^{-1} \mathrm{~m}^{-1}$, a gaussian input pulse with $P_{0}=1 \mathrm{~kW}$ and $T_{0}=0.1 \mathrm{ps}$. These parameters lead to $\beta_{3}=0.001 \mathrm{ps}^{2} \mathrm{~m}^{-1}$, and the propagation took place throughout $11 \mathrm{~mm}$. In this case, the theoretical resonance wavelength is $\lambda_{\text {res }}=2311 \mathrm{~nm}$, while the numerical value is $2362 \mathrm{~nm}$, in close agreement with the theoretical prediction.

\section{Analysis considering realistic waveguide designs}

In order to leverage the potential of OWB for supercontinuum generation, one must place careful attention to the design of the dispersion. However, TPA will limit notably the spectral broadening of pulses pumped at telecom wavelengths if new frequencies are entirely generated through SPM [2]. Nevertheless, in [6], we did already point out that the impact of nonlinear losses can be directly reduced by means of a suitable pump pulse profile. In that work we numerically demonstrated that this goal can be achieved by adding a cubic spectral phase, $\eta\left(\omega-\omega_{0}\right)^{3}$, where $\eta$ controls the so-called skewness of the pulse. Based on the approach presented in the previous section and taking into account the pulse skewness, we find a dispersion profile characterized by $\beta_{2}=0.24 \mathrm{ps}^{2} \mathrm{~m}^{-1}, \beta_{3}=0.0012 \mathrm{ps}^{3} \mathrm{~m}^{-1}$ leading to a huge spectral broadening even in presence of TPA. Finally, we determine a suitable waveguide geometry matching the above parameters by means of our inverse dispersion engineering tool [7]. We choose a strip slot waveguide geometry on a SOI platform and obtain the following values for its geometrical parameters: $w=840 \mathrm{~nm}, h=524 \mathrm{~nm}, h_{\mathrm{s}}=39 \mathrm{~nm}$, and $d_{\mathrm{s}}=108 \mathrm{~nm}$, being $w$ and $h$ the width a height of the silicon channel, respectively, and $h_{\mathrm{s}}$ and $d_{\mathrm{s}}$ the height and position of the slot, respectively. The output spectrum of a gaussian input pulse defined by $P_{0}=1 \mathrm{~kW}, T_{0}=0.1 \mathrm{ps}$, and a skewness $\eta=-0.16 T_{0}^{3}$ after $8 \mathrm{~mm}$ propagation length is shown in Fig. 2(a). For this last simulation, TPA, nonlinear coefficient dispersion, free-carrier dispersion and absorption are included. The spectrum spans beyond a $2 / 3$ of an octave, see Fig. 2(a), and is highly coherent across the bandwidth, see Fig. 2(b). The similarity with respect to the case in Fig. 1(b) indicates that resonant OWB plays a major role in the supercontinuum generation.

\section{References}

1. M. Lipson, "Guiding, Modulating, and Emitting Light on Silicon. Challenges and Opportunities", J. Lightwave Technol.23, 4222-4238 (2005).

2. P. Koonath, D. R. Solli, and B. Jalali, "Limiting nature of continuum generation in silicon", Appl. Phys. Lett. 93, 091114 (2008).

3. K. E. Webb, Y. Q. Xu, M. Erkintalo, and S. G. Murdoch, "Generalized dispersive wave emission in nonlinear fibers", Opt. Lett. 38, 151-153 (2013).

4. D. Castelló-Lurbe, P. Andrés, and E. Silvestre, "Dispersion-to-spectrum mapping in nonlinear fibers based on optical wave-breaking", Opt. Express 21, 28550-28558 (2013).

5. M. Conforti and S. Trillo, "Dispersive wave emission from wave breaking", Opt. Lett. 38, 3815-3818 (2013).

6. D. Castelló-Lurbe, E. Silvestre, P. Andrés i V. Torres-Company, "Spectral broadening enhancement in silicon waveguides through pulse shaping", Opt. Lett. 37, 2757-2759 (2012).

7. D. Castello-Lurbe, V. Torres-Company, and E. Silvestre, "Inverse dispersion design in silicon waveguides," in Optical Fiber Communication Conference, OSA Technical Digest (online) (Optical Society of America, 2014), paper Th2A.39. 